\title{
Kinematics of Entrapped Air Pockets within Stormwater Storage Tunnels
}

\author{
José G. Vasconcelos and Carmen D. Chosie
}

During the rapid filling of stormwater storage tunnels, which is anticipated to occur during intense rain events, a number of different mechanisms may lead to the entrapment of air pockets, as described by Vasconcelos and Wright (2006). Entrapped air pockets have been linked to operational issues such as damaging surges, loss of storage capacity and severe geysering upon their release through water filled vertical shafts. However, the mechanisms behind the motion of these finite volume pockets following their entrapment still require further investigation.

For discrete air pockets (as opposed to stratified air-water flow conditions) a balance between drag and buoyancy is expected to control the motion of large pockets in closed conduits. Previous related investigations approached the problem of pocket motion in the context of water mains, focusing on the determination of a minimum velocity to ensure air pocket removal. In the context of the rapid filling of stormwater tunnels, water flows cannot be pre-specified, and the pocket celerity thus becomes an important parameter, particularly in the context of numerical simulation for such flows.

This chapter presents the preliminary results of an experimental investigation performed at Auburn University in which different air pocket volumes are released in pressurized closed pipe flow at selected slopes and flow rates. The kinematics of air pockets are studied for various flow conditions with the goal of obtaining a better understanding between observed pocket celerity and its relation to pocket volume, flow velocity and pipeline slope.

Vasconcelos, J. and C.D. Chosie. 2013. "Kinematics of Entrapped Air Pockets Within Stormwater Storage Tunnels." Journal of Water Management Modeling R246-10. doi: 10.14796/JWMM.R246-10.

(C) CHI 2013 www.chijournal.org ISSN: 2292-6062 (Formerly in Pragmatic Modeling of Urban Water Systems. ISBN: 978-0-9808853-8-5) 


\subsection{Introduction}

Deep storage tunnels have been utilized in highly urbanized areas as the means to provide relief to the stormwater collection systems as well as treatment to runoff. Operational problems in these systems, such as damaging surges and geysering episodes, has led to investigations aimed at identifying the causes of these problems, which have been observed when tunnels undergo rapid filling during intense rain events. Recent investigation has been helpful in identifying the role of entrapped air pockets in these flows. There is evidence that entrapped air pockets have major impacts in system behavior, such as pressure surge magnitudes, storage capacity loss, and geysering. A better understanding of the air pocket behavior in these tunnels is needed so that better design guidelines can be created to avoid these adverse conditions.

One of the most extreme examples of the adverse interactions between stormwater and entrapped air phases is the occurrence of urban geysering. Figure 10.1 presents images of recent geyser episodes recorded in urban areas as result of intense rain events. The images suggest a variety of public health and safety hazards, including the release of poor quality water to the ground surface and the flooding of major roadways. Evidence of the relationship of geysering and the rapid filling of closed conduits may be found in works by Guo and Song (1991), Nielsen and Davis (2009) and Wright et al. (2011).

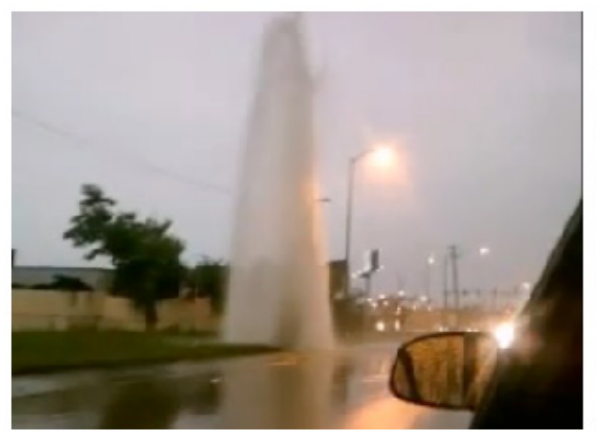

(A)

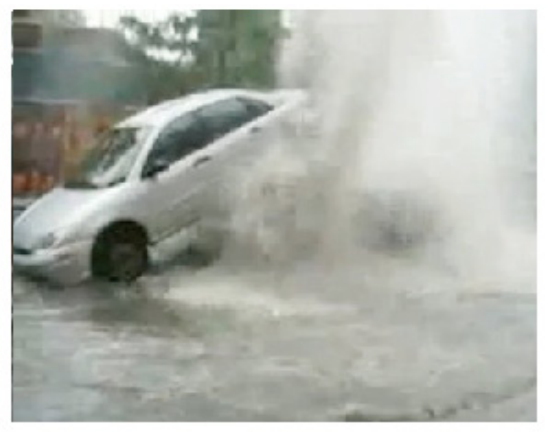

(B)

Figure 10.1 Different urban geyser incidents: (a) image from geyser video, reportedly recorded at Chicago, IL on 201006 23; (b) geyser lifting a car in Montreal, Quebec on 20110718.

The initial theoretical framework proposed to investigate geysers by Guo and Song (1991) was based on inertial oscillations of the water mass within the conduits. This framework states that when the hydraulic grade line 
(HGL) is above grade geysers will occur. Yet evidence presented by Wright et al. (2011) of field pressure measurements during an actual geyser episode shows that such episodes occurred even when the HGL was far below the grade. Also, experimental work by Vasconcelos and Wright (2011) has proven that the release of large air pockets through water-filled ventilation towers may lead to geysering, and the likelihood of such events increases significantly for smaller diameter ventilation towers.

Other relevant studies on the pressure surges generated by entrapped air pockets during unsteady flows are exemplified by the works of Zhou et al. (2002) and De Martino et al. (2008), which are founded on the earlier investigation of Martin (1976). As these works indicate, a compressed air pressure head may exceed many times the initial water pressure that generated the flow, which in turn could damage stormwater system components.

The inclusion of air phase modeling in hydraulic models should result in a better representation of the flow conditions anticipated in stormwater systems during intense rain events. To achieve this goal, the first step is an understanding of the mechanisms leading to pocket formation. Vasconcelos and Wright (2006) summarized some of the known mechanisms leading to these entrapments in the context of stormwater system flows, including, among other mechanisms, surface waves created by the relative motion of air and water, lack of adequate ventilation and interface breakdown phenomena. Figure 10.2 overleaf presents an air pocket formation mechanism observed in experiments where the reflection of an inflow front from a system boundary entraps an air pocket in this location. Figure 10.3 overleaf indicates the same occurrence, but this time observed in numerical modeling of the rapid filling of a stormwater tunnel presented by Vasconcelos and Leite (2011). Other studies related to the formation and motion of air in stormwater systems are exemplified by Hamam and McCorquodale (1982), Li and McCorquodale (1999), Zhou et al. (2002) and Lautenbach et al. (2008).

The next step in the incorporation of air effects in numerical modeling requires a description of discrete air pocket motion within pressurized water flows. This area has been studied in different contexts to date. Falvey (1980), Pozos et al. (2010), Pothof and Clemens (2010) and others have studied the motion of air pockets and bubbles in pressurized mains with the objective of deriving an expression for the minimum water velocity that ensures air pocket removal from pipes (clearing velocity). The quasi-steady motion of discrete air pockets in pressurized pipe flows has been investigated in the context of multi-phase flow applications (DeHenau and Raithby, 1995). These slug flows are caused by steady injection of gaseous and liquid flows at one end of a pipeline, a condition that is often observed in petroleum engineering. 


\section{$\mathrm{T}=\mathrm{T} 1$}
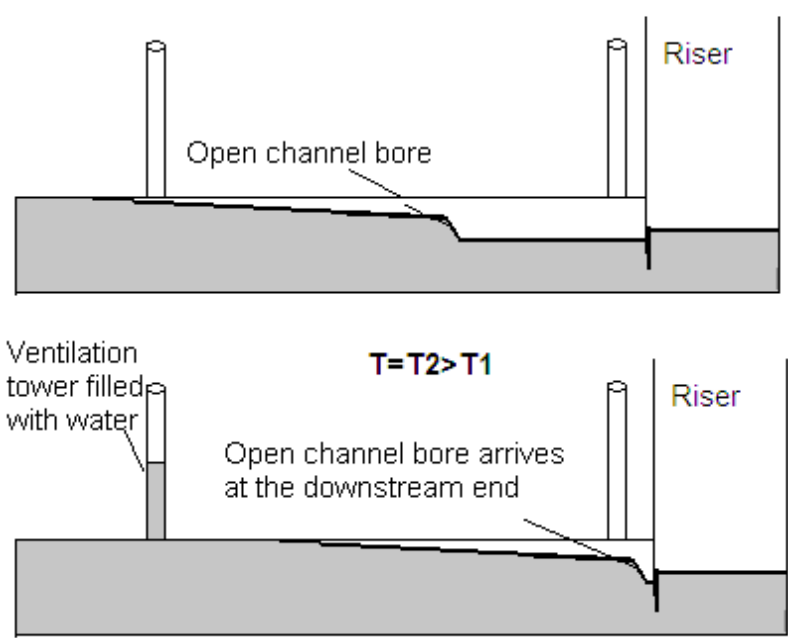

Ventilation tower filleds with water

$T=T 3>T 2$

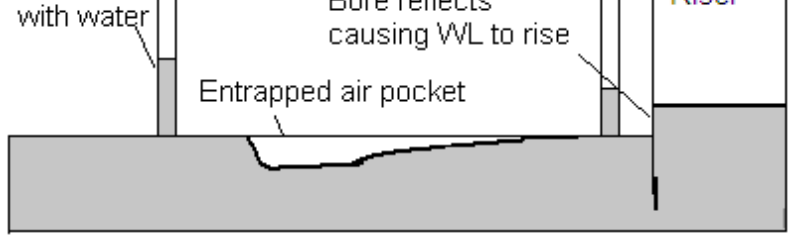

Figure 10.2 Air pocket entrapment mechanism related to the reflection of inflow fronts from the system boundary (Vasconcelos and Wright, 2006).

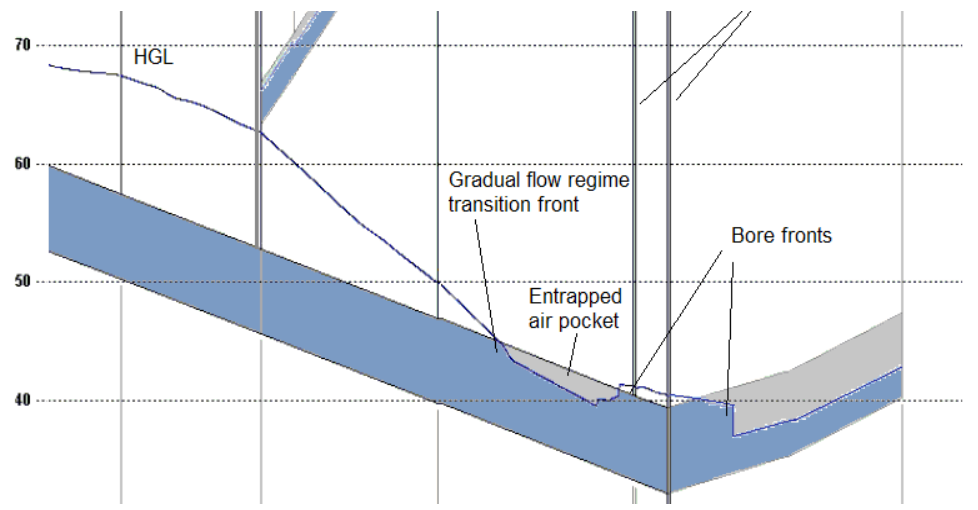

Figure 10.3 Air pocket entrapment mechanism related to the reflection of inflow fronts from the system boundary (Vasconcelos and Leite, 2011). 
The motion of discrete air pockets has also been studied along with gravity currents on slope (Baines, 1991), and a general expression for the pocket's celerity can be expressed as:

$$
C(h)=k(h) \sqrt{g h}
$$

where:

$C=$ air pocket celerity $(\mathrm{m} / \mathrm{s})$,

$k=\mathrm{a}$ factor that depends on the pocket thickness,

$h=$ current thickness (m), and

$g=$ gravitational acceleration $\left(\mathrm{m} / \mathrm{s}^{2}\right)$.

More recently, Glauser and Wickenhauser (2009) presented a relevant work that includes a study of the motion of air pockets in a downward inclined experimental apparatus. The authors observed the existence of a critical air pocket volume for a certain water flow rate and slope. Below this volume threshold the pocket would be dragged by the flow against buoyancy, but this volume also depended on the pipeline slope as it affected the shape of the air pocket. However, in their study, the tested slopes included only values between $1.7 \%$ and $8.7 \%$, much higher than those generally encountered in stormwater storage tunnels. Moreover, the slopes tested were only favorable (when drag caused by the water flow opposes air pocket buoyancy), and thus expressions for the air pocket involving the combination of these two forces were not presented.

\subsection{Objectives}

This chapter presents ongoing research for which the main objective is to establish a link between flow velocity, pipeline slope and the celerity of entrapped air pockets of various volumes.

The focus is to present a discussion on the kinematics of entrapped air pockets in pressurized water flows under shallow slopes. These preliminary results will support the improvement of numerical modeling of entrapped air pockets within stormwater storage tunnels. These will include the trajectory of entrapped air pockets upon sudden release at an intermediate point in the experimental apparatus described in the following Methodology section. Such release is just a means to introduce a known air pocket volume in a pressurized pipe flow. A brief, qualitative description of the air pocket shape is included.

This study also attempts to establish a link between the observed air pocket celerity with theoretical predictions of pocket celerity and the flow velocity. 


\subsection{Methodology}

Figure 10.4 presents a schematic of the experimental apparatus used in the experiments, which is essentially a variable slope clear PVC pipeline supplied at the upstream end by a fixed head reservoir while having a throttled discharge at the downstream end that ensures a pressurized flow regime in the entire system. The pipeline has an approximate length $L=12 \mathrm{~m}$ and diameter $D=101.6 \mathrm{~mm}$. The upstream supply reservoir is connected to the pipeline by a $50 \mathrm{~mm}$ diameter ball valve. Two $101.6 \mathrm{~mm}$ diameter knife gate valves were positioned $3 \mathrm{~m}$ from the upstream end with a $2.1 \mathrm{~m}$ separation. Pre-determined volumes of air at atmospheric pressure were injected at the region between the valves forming an air pocket, but the partially-closed gates prevented the motion of these pockets while allowing water flow under it.

Air pockets of different volumes were supplied by a graduated acrylic air tank located at the bottom of the water pipeline, which was separated from the PVC pipeline by a control valve. Valves at this supply tank ensured atmospheric pressure for the supplied air prior to the opening of the control valve that allowed the admittance of air into the region between the two partially closed knife-gate valves.

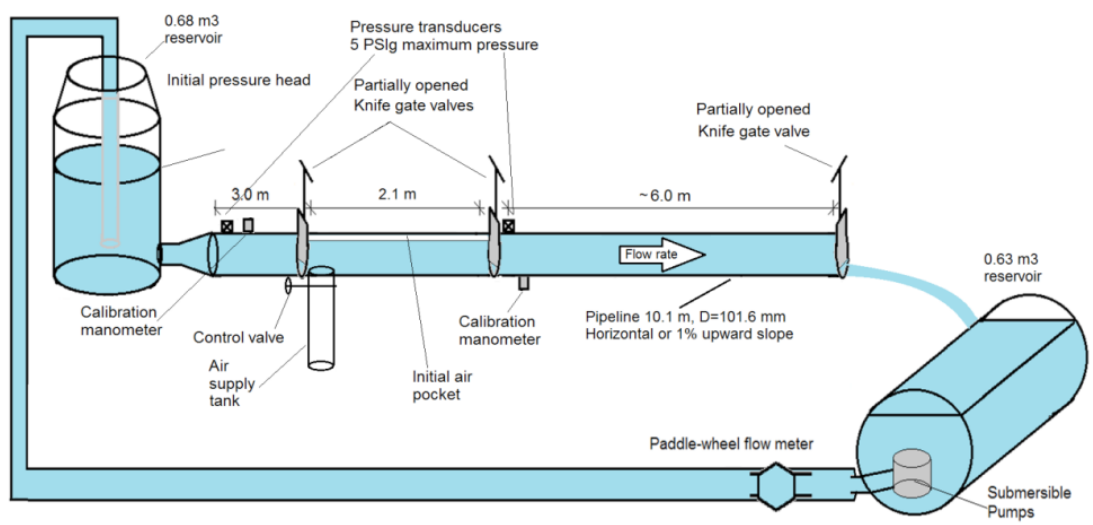

Figure 10.4 Sketch of the experimental apparatus used in the experiments.

The measurement devices used in this experiment included:

- up to four high definition camcorders (1080 pixels, $30 \mathrm{fps}$ ), providing a combined field of view of $7.0 \mathrm{~m}$ upstream and downstream from the intermediate valves;

- two piezoresistive pressure transducers, Meggit Endevco $8510 \mathrm{~B}-5$, located at the upstream end $\left(X^{*}=X / L=0.0\right)$ and at 
approximately $50 \%$ of the pipeline length $\left(X^{*}=0.5\right)$, recording with a frequency of $100 \mathrm{~Hz}$ for each channel; and

- National Instruments data acquisition board NI-USB 6210 with SignalExpress logging software.

The experimental procedure may be described as following:

1. The selected pipeline slope was set in the apparatus;

2. Steady flow conditions were created at the clear PVC pipeline by starting up the submersible pumps at the desired flow rate and setting up the opening of intermediate knife gate valves;

3. Air volume at atmospheric pressure was selected at the supply tank and then released into the system by opening the tank's control valve;

4. The system was allowed to achieve a new steady flow as air addition in some cases resulted in increased energy losses;

5. Pressure measurement was initiated and readings on calibration manometers were performed;

6. Rapid opening $(t<0.6 \mathrm{~s})$ of the intermediate knife gate valves led to the sudden release of the air pocket;

7. Camcorders recorded the motion and spreading of the air pockets until they moved outside the combined field of view; and

8. Valves were closed and after some time pumps were stopped and final readings on the calibration manometers were performed.

The preliminary set of results presented in this chapter includes more than 40 different conditions. Four slopes were tested to date: horizontal slope, and $0.5 \%, 1.0 \%$ and $2.0 \%$ upward slope (downstream end at a higher elevation than inlet). For each slope three different flow rates were tested, ranging from $1.4 \mathrm{~L} / \mathrm{s}$ up to $4.0 \mathrm{~L} / \mathrm{s}\left(Q^{*}=Q / \sqrt{ } g D^{5}=0.136\right.$ to 0.388$)$. For each combination of slope and flow rates, up to four different initial air pocket volumes were tested. For horizontal slopes these volumes ranged between $1.3 \mathrm{~L}$ and 4.3 L $\left(\mathrm{Vol}^{*}=\mathrm{Vol} / \mathrm{D}^{3}=1.2\right.$ to 4.1), while for upward slope experiments these volumes ranged between $0.5 \mathrm{~L}$ and $4.0 \mathrm{~L}\left(\mathrm{Vol}^{*}=0.48\right.$ to 3.8$)$. One criterion for the selection of air pocket volumes was to avoid air being dragged by the flow under the gate valves and escaping downstream prior to the valve opening. It was observed that this drag was more pronounced for larger flow rates, and therefore it was not possible to conduct some runs with larger air pocket volumes and flow rates. In addition to the conditions described above, experiments included the study of the pocket thinning when a single gate was opened in conditions of no flow and horizontal slope.

The monitoring of the pressures in the system had three main objectives: to gain insight of what the pressure variation was during the motion of the 
released pockets; to assess the amount of energy loss caused by the partially closed valves; and to measure the thickness of air pockets at selected cases (since there is evidence in the literature of the relationship between air pocket thickness and its celerity during its motion, which in this instance is caused by drag or buoyancy forces). All cases were repeated at least once to ensure the consistency of the data collected. Table 10.1 presents a summary of the parameters tested so far in this investigation.

Table 10.1 Experimental variables used in the experiments with respective ranges.

\begin{tabular}{ll}
\hline \multicolumn{1}{c}{ Parameter } & \multicolumn{1}{c}{ Range Tested } \\
\hline Flow rate (normalized by $\left.Q / \sqrt{ } g D^{5}\right)$ & Three values ranging from $Q^{*}=0.136$ up to 0.388 \\
Pipeline slope & Horizontal, $0.5 \%, 1.0 \%$ and $2.0 \%$ upward \\
& Horizontal slope: up to 4 values between $\mathrm{Vol}^{*}=1.2$ and 4.1 \\
Air pocket volume (normalized by $\left.D^{3}\right)$ & Upward slopes: up to 4 values between $\mathrm{Vol}^{*}=0.48$ and 3.8 \\
\hline
\end{tabular}

${ }^{1}$ Pipeline downstream end elevation higher than upstream

\subsection{Results and Discussion}

\subsubsection{Trajectory of the Released Water Pockets}

The first type of experiment presented involves a release of an air pocket in a horizontal pipeline with no flow. This simple spreading condition resembles the flow of a gravity current with limited volume. The data collected regarding the air pocket front trajectories and respective celerity values is presented in Figure 10.5. The higher celerity values observed in conditions involving larger air pocket volumes is consistent to previous investigations by Benjamin (1968) and Wilkinson (1982). Measured celerity values were smaller than theoretical predictions by Benjamin or Wilkinson, with the latter providing closer agreement.

An interesting feature shown in Figure 10.5 is the air pocket spreading that occurs in horizontal conditions. This spreading affects the thickness of the advancing front, which in turn will result in smaller pocket celerity over time, as shown in Figure 10.6b; correspondingly, pocket front trajectories in Figure 10.5 are curved. Camera recordings of the advance of air pockets toward the upstream end indicate that the trajectory of this back propagating front is symmetrical to the forward propagating front (toward downstream) in the absence of flow in the pipe, as would be anticipated. This is reflected in the symmetric trajectory of the back propagating front in Figure 10.6a. Because the spreading when two valves are opened (double direction) is more pronounced than when a single valve is opened, steeper trajectories (slower front motion) for double spread cases are observed in Figure 10.5. 


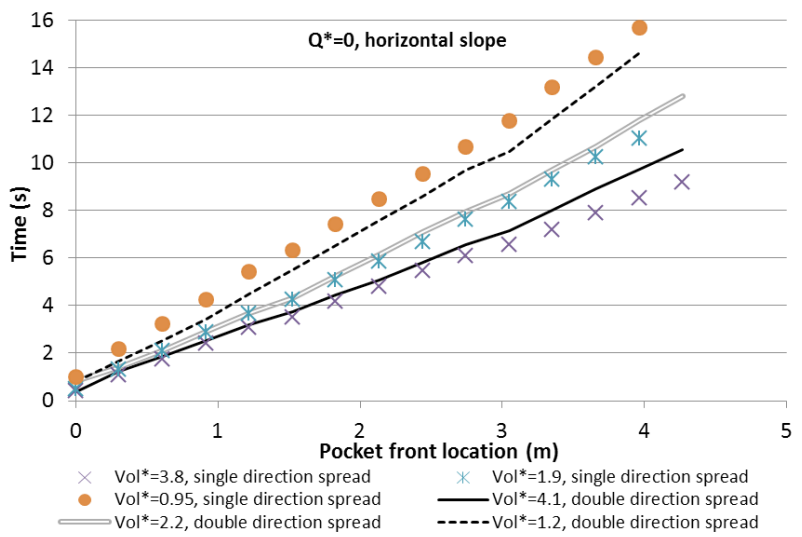

Figure 10.5 Trajectory of air pocket front during simple spreading experiments.
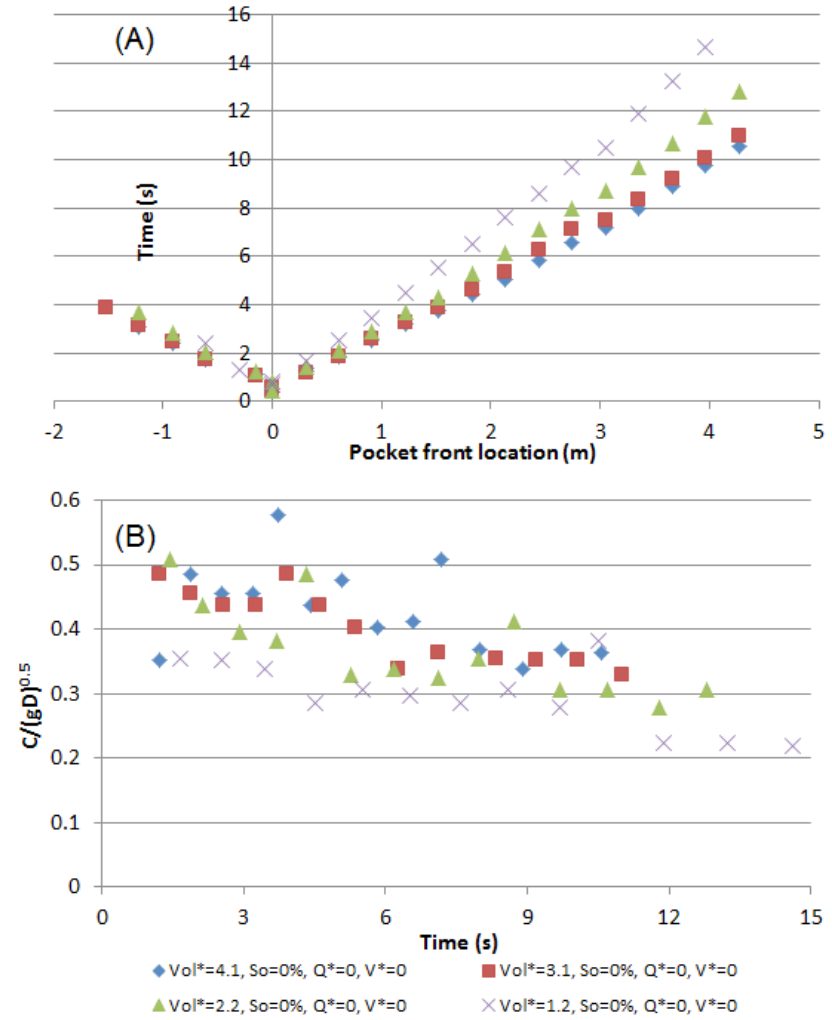

Figure 10.6 Pocket trajectory (a), and dimensionless celerity (b), for experiments with horizontal slope, various air pocket volumes and no flow in the system; ambient velocity is normalized as $V^{*}=(Q / A) / \sqrt{ } g D$. 
Figure 10.7 presents a comparison of the trajectories of the air pocket front upon the opening of the gates for the horizontal and the three adverse slopes tested. In these charts there is no net water flow. The results indicate that the curved trajectory of the air pockets in the horizontal case is not observed in the adverse slope case, even for the smallest slope tested of $0.5 \%$. These results are aligned with visual observations of the pocket motion that indicated the spreading seen in horizontal pocket propagation is smaller for sloped experiments as air is concentrated at the pocket front. A second observation is that the relative difference between the pocket celerity, which can be inferred by the trajectory slope, becomes smaller for larger pipe slopes. This is an indication of the concentration of air at the nose for larger adverse slopes, rendering the thickness differences smaller, which explains the similar celerity values. A third observation is that for all tested cases, the non-dimensional celerity values $c^{*}=c / \sqrt{ } g D$ is $<0.467$, which is consistent with similar measurements presented by Baines (1991) for the propagation of air cavities in sloped, squared, pipe cross sections. The much slower motion for the case with smallest volume $V_{o} l^{*}=0.48$ and $2 \%$ adverse slope cannot be explained at this time, but it is speculated that it may be due to the effects of friction.
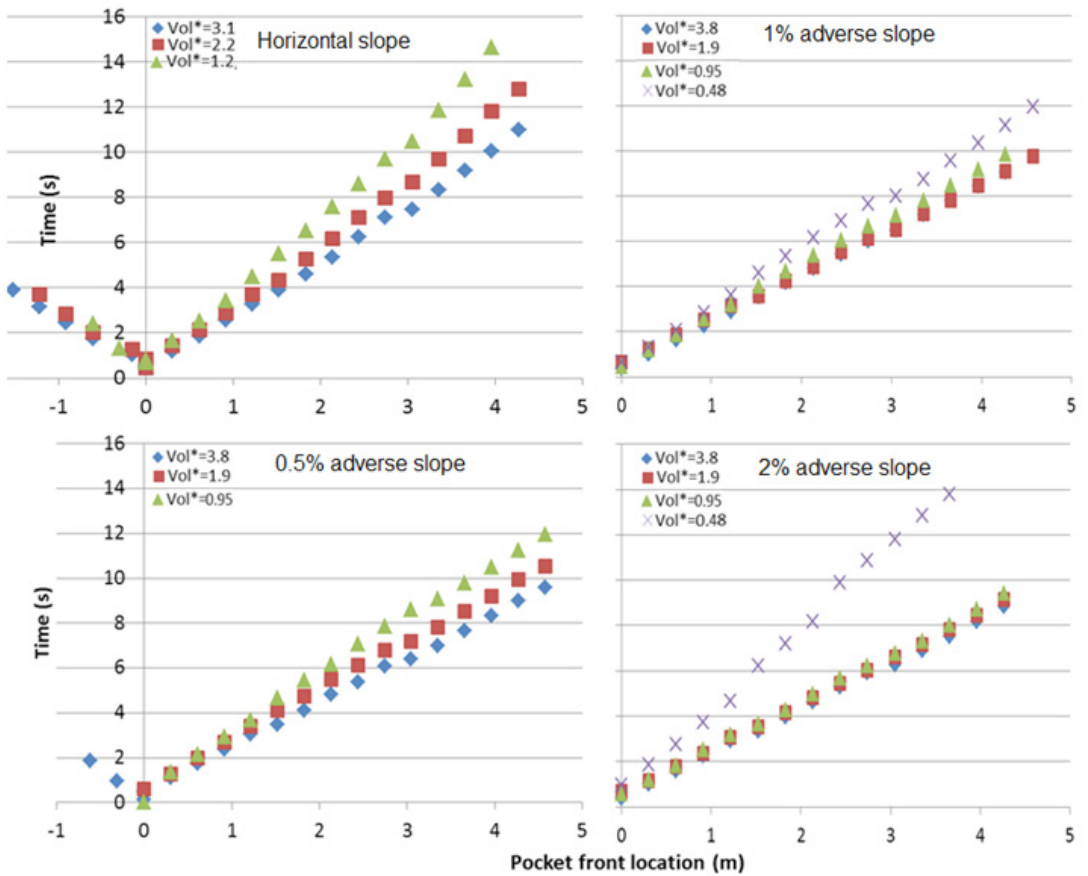

Figure 10.7 Pocket trajectory for various slopes and $\mathrm{Vol}^{*}=3.1$ and 4.1 , with no water flows. 
To study the case of the motion of air pockets due to the water flow, it was decided first to consider the horizontal case. Figure 10.8 overleaf presents trajectory and dimensionless celerity results for the case when $\mathrm{Vol}^{*}=$ 2.2 and for various flow rates. Figure 10.8a indicates that the once observed symmetry of the pocket propagation in two directions for horizontal flow conditions is no longer observed. The propagation of the air pocket against the flow of $Q^{*}=0.13$ (non-dimensional velocity $V^{*}=V / \sqrt{ } g D=0.17$ ) occurs for a length of approximately $3 \mathrm{~m}$ from the initial position of air pocket release, after which the shearing of the water flow erodes the back propagating air pocket front; this is further discussed later in this chapter. In this process the pocket celerity is reduced to approximately $C^{*}=-0.25$. Back propagation of the air pocket front is not observed for larger flow rates as the pocket shearing occurs much more rapidly. Drag forces also play a role in retarding the back propagation of the front.

As is shown in Figures $10.8 \mathrm{a}$ and $10.8 \mathrm{~b}$, weaker air pocket front trajectories (larger celerities) are observed for larger water flows, an expected result. All cases presented in the figure were for a common air pocket volume of $V_{o l}{ }^{*}=2.2$. The observed air pocket front thinning in the horizontal, no flow, case, which leads to decreasing celerity values, is not as pronounced for the cases when there is water flow. Finally, an interesting observation when the flow velocity is subtracted from the observed pocket celerity was made, as is shown in Figure 10.8c. One notices that the remainder value is not a monatomic function of the flow rate. We speculate that this result could be linked to a change in the air pocket front shape caused by flows with higher velocity. These findings may be summarized as follows:

1. As a first approximation, based on the results obtained in the tested experimental scale, the co-current air pocket front celerity may be assumed to be the celerity of the front in quiescent conditions plus the water flow velocity;

2. This assumption may lead to over-estimated celerity values in cases when the water velocity is in the large tested range (i.e. $V^{*} \approx 0.49$ ); and

3. The propagation of air pockets against water flow, even if the flow velocity is less than half of the pocket celerity, will be short lived due to shear effects eroding the air pocket front.

However interesting these findings, the scale effects of air and water flows are not completely understood, and thus planned future investigation will include the use of larger clear PVC pipeline diameters $(\sim 200 \mathrm{~mm})$ and smaller diameters $(\sim 50 \mathrm{~mm})$ to verify these observations. 


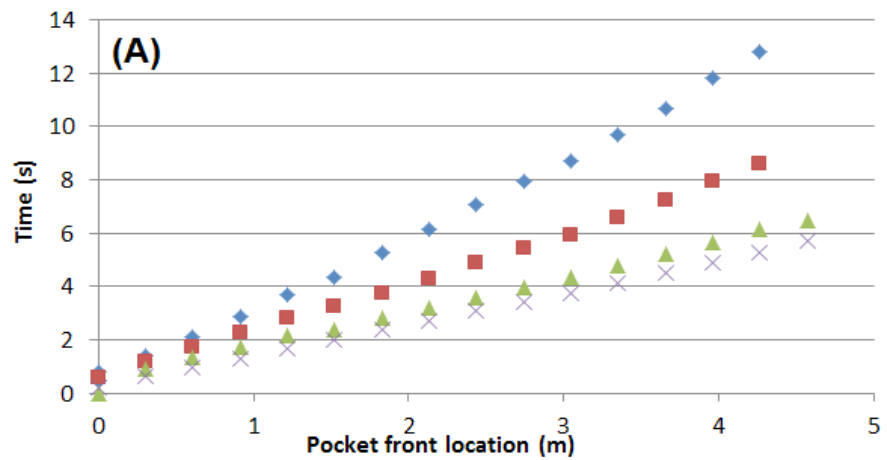

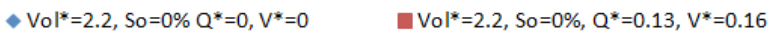

$\triangle \mathrm{Vol}^{*}=2.2, \mathrm{So}=0 \%, \mathrm{Q}^{*}=0.27, \mathrm{~V}^{*}=0.34 \times \mathrm{Vol}^{*}=2.2, \mathrm{So}=0 \%, \mathrm{Q}^{*}=0.39, \mathrm{~V}^{*}=0.49$
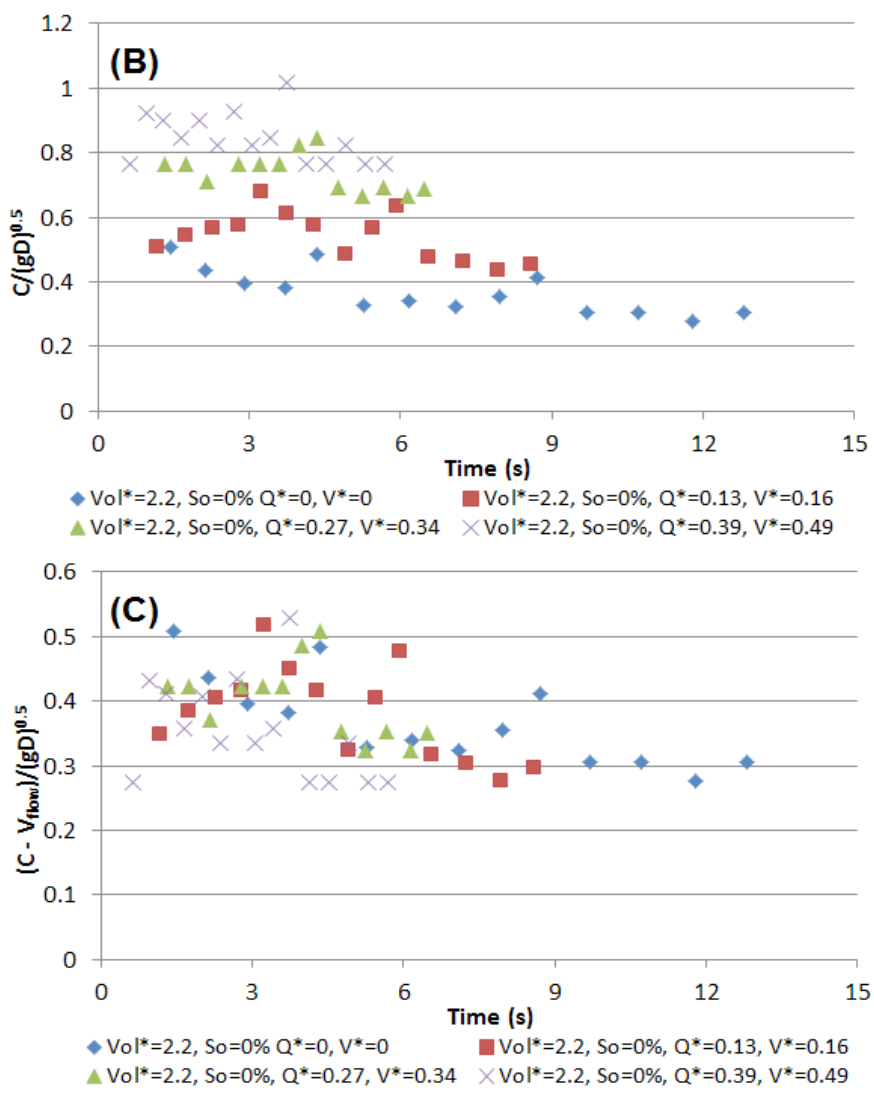

Figure 10.8 Pocket trajectory (a), dimensionless celerity (b), and dimensionless celerity minus flow velocity (c), for experiments with horizontal slope, fixed air pocket volume $\left(\mathrm{Vol}^{*}=\right.$ 2.2) and various flow rates. 

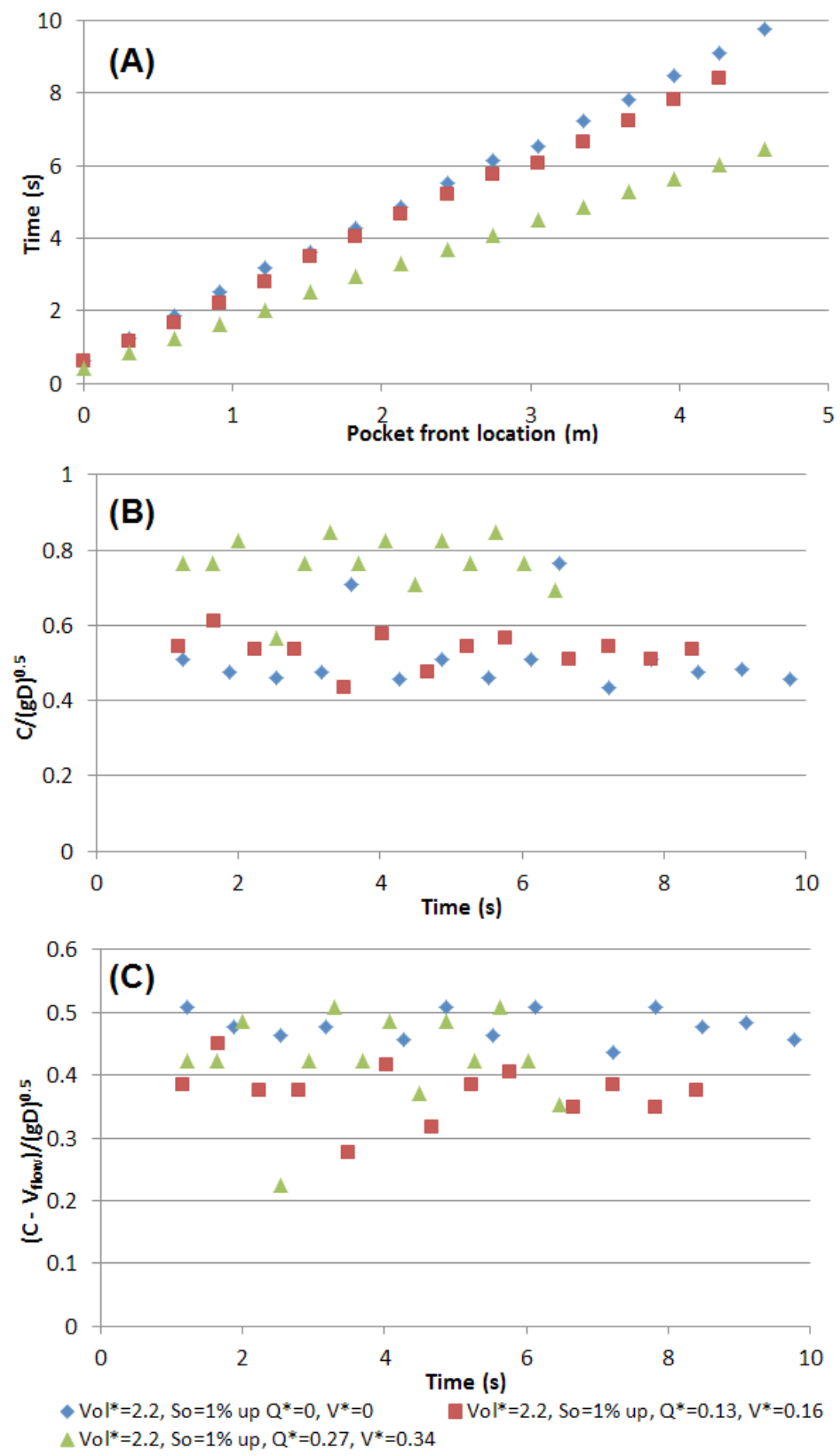

Figure 10.9 Pocket trajectory (a), dimensionless celerity (b), and dimensionless celerity minus flow velocity (c), for experiments with $1 \%$ upward slope, fixed air pocket volume $\left(\mathrm{Vol}^{*}\right.$ $=2.2$ ) and various flow rates. 


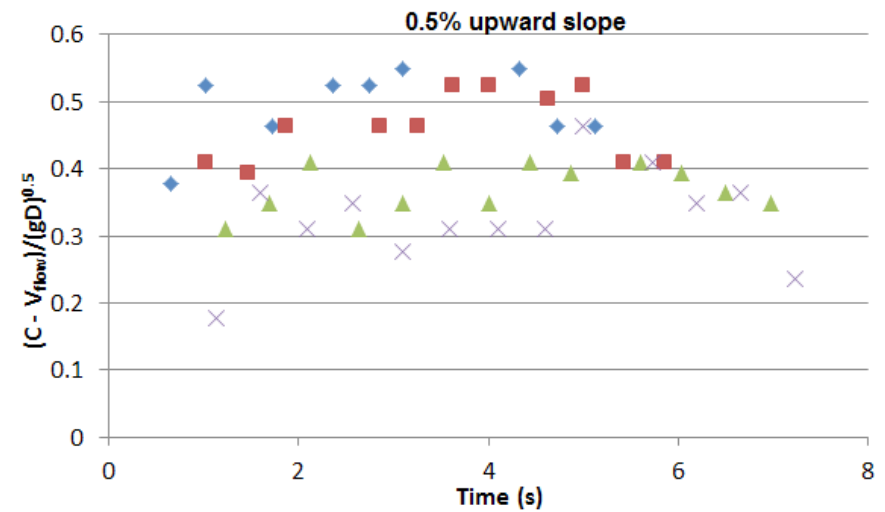

- Vol ${ }^{*}=2.9$, So $=0.5 \%$ up, $\mathrm{Q}^{*}=0.27, \mathrm{~V}^{*}=0.34 \quad \square \mathrm{Vol}^{*}=1.9$, So $=0.5 \%$ up, $\mathrm{Q}^{*}=0.27, \mathrm{~V}^{*}=0.34$ $\triangle \mathrm{Vol}^{*}=0.95$, So $=0.5 \%$ up, $\mathrm{Q}^{*}=0.27, \mathrm{~V}^{*}=0.34 \times \mathrm{Vol}^{*}=0.48$, So $=0.5 \%$ up, $\mathrm{Q}^{*}=0.27, \mathrm{~V}^{*}=0.34$

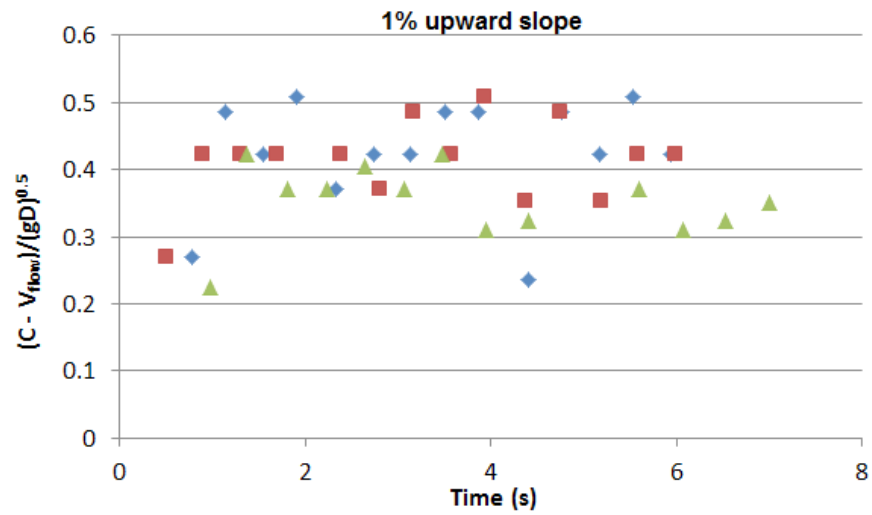

$\Delta V_{0}^{*}=1.9$, So $=1 \%, Q^{*}=0.27, V^{*}=0.34$

$\triangle V_{0} I^{*}=0.49$, So $=1 \%, Q^{*}=0.27, V^{*}=0.34$

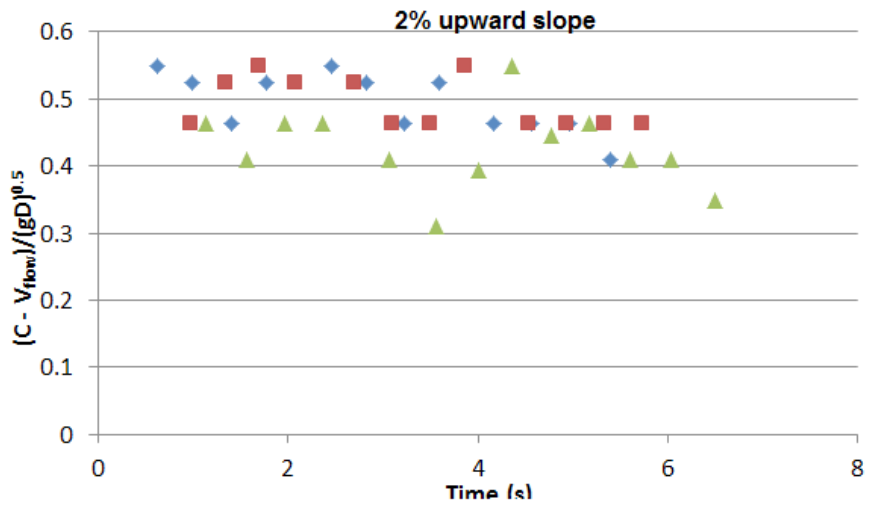

- $\mathrm{Vo}^{*}=1.4$, So $=1 \%, \mathrm{Q}^{*}=0.27, \mathrm{~V}^{*}=0.34$

$\triangle V_{0} l^{*}=0.49$, So $=1 \%, Q^{*}=0.27, V^{*}=0.34$

Figure 10.10 Pocket front relative celerity for experiments with upward slopes, various air pocket volumes and flow rate $Q^{*}=0.27$. 
Figure 10.9 above is similar to Figure 10.8, but this time the intention is to study the effects of flow velocity in sloped conditions ( $1 \%$ upward). As anticipated, the observed air pocket front celerity increases with the flow rate. There is no significant difference in the celerity values between the no flow case and when the smallest flow rate $Q^{*}=0.13$ was used; this difference becomes more significant for the case when $Q^{*}=0.27$. The relative celerity (observed minus ambient flow velocity $V=Q / A$ ) is comparatively similar between the presented cases, albeit there is a somewhat larger scatter in the results obtained within cases when there was ambient flow.

Figure 10.10 opposite presents measurements of the observed air pocket front celerity minus the flow velocity for various slopes and air pocket volumes, when the ambient flow was $Q^{*}=0.27$. It represents the combination of varying upward slopes and initial air volumes, both factors that have minimized the effect of air pocket spreading in the decrease of the air front thickness, and thus a decay of the pocket front celerity. The results indicate that larger air pocket volumes still result in larger pocket celerities, though this difference becomes less pronounced for larger upward slopes. The relative celerity of the pocket front for the smallest air volume increases from an average of 0.3 for the $0.5 \%$ upward slope to a value $>0.4$ for the upward slope of $2.0 \%$. On the other hand, the average relative celerity for the largest air pocket front for the largest air volumes had a small variation between the tested slopes.

\subsubsection{Shearing of Air Pocket Front Propagation Against the Flow}

An interesting observation in the experiments performed with horizontal slope and positive values for the flow rate was the change in the shape of the back-propagating front, which moved against the pipe flow. Figure 10.11 presents seven snapshots of the pocket propagation with $1 \mathrm{~s}$ time lapse between the images, for a condition corresponding to a pocket with $\mathrm{Vol}^{*}=4.1$ and flow rate $Q^{*}=0.27$. The initial shape of the front resembles a typical, dissipative gravity current with a curved nose followed by a hydraulic jump, as described in Benjamin (1968). As the front moved upstream, the hydraulic jump that followed it entrained the air of the pocket, and these smaller pockets were carried with the flow. The size (thickness and length) of the pocket decreased over time, and it eventually disappeared. In the process, the celerity of the pocket decreased gradually as the nose size decreased, which is consistent with theory. The other extremity of the front did propagate as a curved gravity current front. We speculate that this type of pocket shearing may also be observed in actual deep stormwater storage tunnels, considering the small slopes that are frequently employed in their design. This behavior was not observed for the cases with upward slopes. 


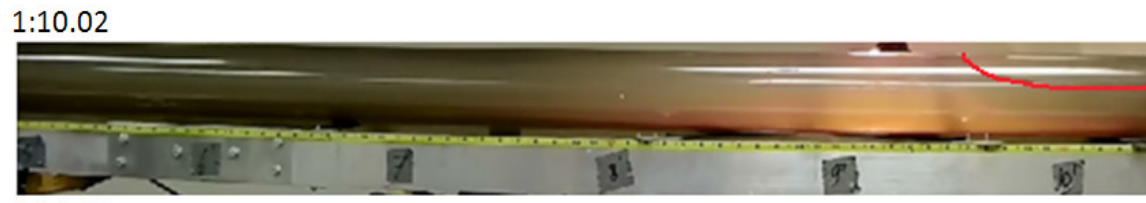

1:11.02

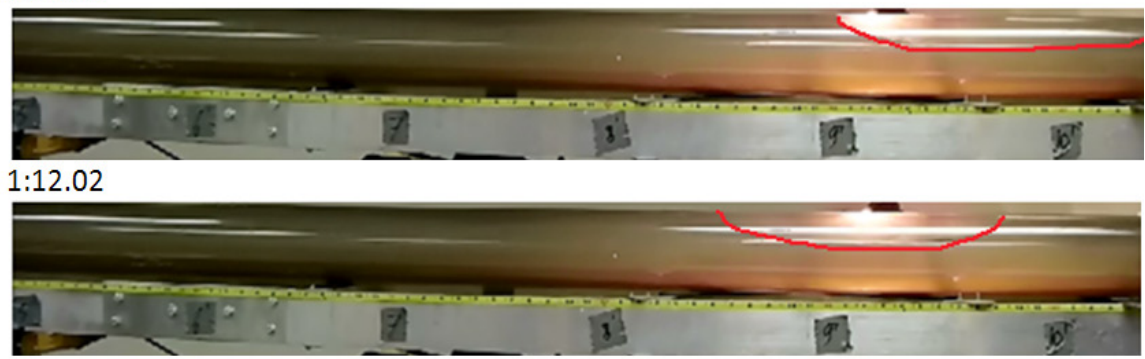

1:13.02

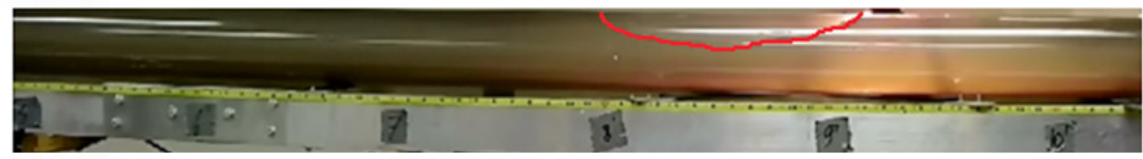

1:14.02

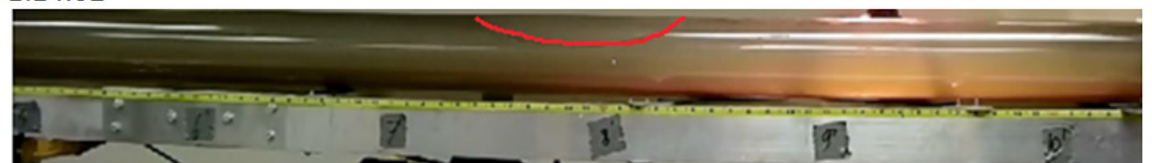

1:15.02

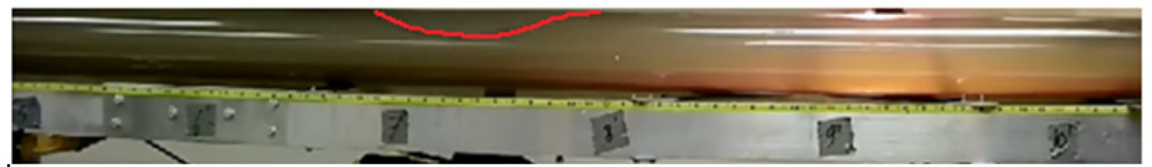

$1: 16.02$

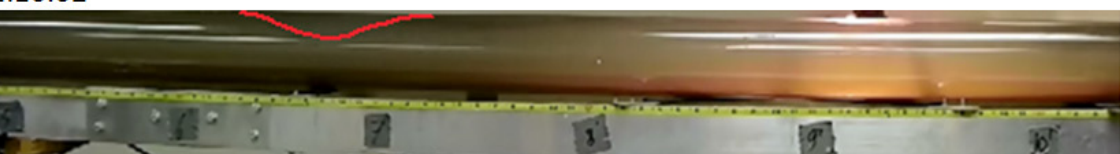

Figure 10.11 Sequence snapshots of pocket trajectory for horizontal slope, no flow, and $\mathrm{Vol}^{*}=2.2$.

The pressure measurements were used to assess whether gate maneuver would causes changes to system pressure, but no significant changes were detected. These pressure measurements can also be useful in determining the air pocket thickness. However, up to this moment, these measurements have not been used to calculate such thickness values. 


\subsection{Summary and Future Work}

As mentioned earlier, this work presents the preliminary results of an ongoing investigation of the motion of entrapped air pockets in pressurized water flow. An important difference from the present investigation and prior work is that there is a focus on air pocket kinematics, so that this information can be properly incorporated in the formulation of numerical models that aim to simulate the dynamics of the rapid filling of stormwater storage tunnels. There is also interest in observing the flow features related to the pocket propagation and the interaction between air and water phases during the pocket motion.

As would be anticipated, the pocket celerity increased with the size of the pockets, and for comparable conditions, experiments with upward slope resulted in higher celerity values. Considering the finite volume of the air pockets, experimental runs with horizontal slope resulted in a pocket spreading that corresponds to a decrease in the pocket celerity with time, a condition that was not observed for upward slope cases. When inflows were introduced into the system, observed celerity increased, and one objective was to assess whether the observed celerity was the summation of the celerity for the case without flows plus the flow velocity. While this seems to be the case for most of the tested cases, for higher flow rates $\left(Q^{*}=0.39\right)$ the increase in the relative celerity was not as high.

These findings may be summarized as follows:

as a first approximation, based on the results obtained in the tested experimental scale, the air pocket front celerity may be assumed as the celerity of the front in quiescent conditions plus the water flow velocity;

this assumption may lead to overestimated celerity values in cases when the water velocity is in the large tested range (e.g. $V^{*} \approx 0.51$ or more); and

the propagation of air pockets against water flow, even if the flow velocity is less than half of the pocket celerity, will be short lived due to shear effects eroding the air pocket front.

However interesting these findings, scale effects of air and water flows are not completely understood, thus planned future investigation will include the use of larger clear PVC pipeline diameters $(\sim 200 \mathrm{~mm})$ and smaller diameters $(\sim 50 \mathrm{~mm})$ to verify these observations.

Future experiments will include more slopes $(0.5 \%, 1 \%$ and $2 \%$ downward slopes), and other pipeline diameters $(50 \mathrm{~mm}$ and $200 \mathrm{~mm})$ will be tested to assess to what extent are the findings are affected by the scale in which the experiments are conducted. 


\section{Acknowledgments}

The authors would like to acknowledge the support provided by LimnoTech Inc., and by Auburn University, which have funded part of this research.

\section{References}

Baines, D. (1991) “Air cavities as gravity currents on slope”. J. Hydr. Engrg., 117(12):16001615.

Benjamin, T. B. (1968). "Gravity currents and related phenomena". J. Fluid Mech. 31, 209-248.

DeHenau, V. and Raithby, G. D. (1995). Transient two-fluid model for the simulation of slug flow in pipelines. Int. J. Multiphase Flow 21 (3), 335-349.

De Martino, G., Fontana, N. and Giugni, M. (2008). "Transient Flow Caused by Air Expulsion through an Orifice” ". J. Hydr. Engrg., 134(9): 1395-1399.

Falvey, H.T. (1980). "Air-water flow in hydraulic structures.” Engineering Monograph No. 41, United States Department of the Interior, Water and Power Resources Service. Denver, CO.

Glauser, S. and Wickenhauser, M. (2009). "Bubble Movement in Downward-Inclined Pipes”. J. Hydr. Engrg., 135(11):1012-1015.

Guo, Q. and Song, C. S. S. (1991). "Dropshaft hydrodynamics under transient conditions." J. Hydr. Engng., 117(8), 1042-1055.

Hamam, M. A. and McCorquodale, J. A. (1982). "Transient conditions in the transition from gravity to surcharged sewer flow." Can. J. Civ. Engrg., (9),189-196.

Lautenbach, D. J., Vasconcelos, J. G., Wright, S. J., Wolfe, J. R., Cassidy, J. F. and Klaver, P. R. (2008). "Analysis of Transient Surge in the Proposed District of Columbia Water and Sewer Authority Deep Tunnel System” Proc. 2008 WEF Collection Systems Conference, Pittsburgh, PA.

Li J. and McCorquodale, A. (1999). "Modeling mixed flow in storm sewers". J. Hydr. Engrg., 125(11):1170-1180.

Martin, C. S. (1976). "Entrapped air in pipelines". Proc. 2nd Int. Conf. Pressure Surges. BHRA Bedford, England.

Nielsen, K. D. and Davis, A. L. (2009). "Air migration analysis of the Terror Lake tunnel," Proc., 33rd IAHR Congress, IAHR, Vancouver, BC, 262-268.

Pothof, I. and Clemens, F. (2010). "On elongated air pockets in downward sloping pipes" J. Hydr. Res. 48(4):499-503.

Pozos, O., Gonzalez, C. A., Giesecke, J., Marx, W. and Rodal, E. A. (2010). “Air entrapped in gravity pipeline systems", J. Hydr. Res. 48(3): 338 - 347.

Vasconcelos, J. G. and Leite, G. M. (2011). "Pressure surges generated by entrapped air pockets in stormwater systems". Proc. 2011 World Water and Environmental Resources Congress ASCE, Palm Springs, CA.

Vasconcelos J. G. and S. J. Wright, S. J. (2006). "Mechanisms for Air pocket Entrapment in Stormwater Storage Tunnels" Proc. 2006 ASCE EWRI Congress, Omaha. 
Vasconcelos J. G. and S. J. Wright, S. J. (2011). "Geysering Generated by Large Air Pockets Released through Water-Filled Ventilation Shafts” J. Hydr. Engrg. Vol. 137(1) $112-115$.

Wilkinson, D. L. (1982). “ Motion of air cavities in long horizontal ducts” . J. Fluid Mech. 118, 109-122.

Wright, S.J., Lewis, J. W. and Vasconcelos, J.G., (2011). “Geysering in Rapidly Filling Storm-Water Tunnels". J. Hydr. Engrg. Vol. 137(5) 543-555

Zhou, F., Hicks, F. E. and Steffler P. M. (2002). "Transient flow in a rapidly filling horizontal pipe containing trapped air”. J. Hydr. Engrg., 128(6):625-634. 
